\title{
Desenvolvimento de tecnologias e negócios em um programa de incentivo à inovação ${ }^{1}$
}

\author{
Luciana Paula Reis Doutora em Administração - Universidade Federal de Ouro Preto (UFOP) - Brasil - luciana.preis@gmail.com \\ Carlos Eduardo Guerra Silva Doutor em Administração - Universidade Federal de Minas Gerais (UFMG) - Brasil - carlose@ufmg.br \\ Reynaldo Maia Muniz Doutor em Ciência Política - Faculdade Novos Horizontes - Brasil - reynaldo.muniz@unihorizontes.br \\ June Marques Fernandes Mestre em Engenharia de Produção - Universidade Federal de Ouro Preto (UFOP) - Brasil - june.marques@gmail.com
}

\section{RESUMO}

O artigo analisa o desenvolvimento de tecnologias em ambiente acadêmico e a consubstanciação dessas em negócios tecnológicos, considerando-se os níveis micro, meso e macro organizacionais. Por meio da análise de conteúdo de relatórios do Programa de Incentivo à Inovação (PII), observou-se no nível micro, o foco difuso de alguns pesquisadores, que oscilam entre empreender e licenciar a tecnologia. No nível meso, foram observados os agentes que compõem o campo da inovação tecnológica, cada qual desempenhando funções específicas. Entre clientes, concorrentes, parceiros e fornecedores, os spinoffs acadêmicos operam sob regras de agências reguladoras, bem como apresentam considerável dependência financeira de agências de pesquisa e programas governamentais. No nível macro, foi constatado que disposições legais, econômicas, tecnológicas, sustentáveis e culturais da ambiência se constituem em referências que orientam a atuação dos diversos agentes de inovação. Os resultados deste estudo contribuem para a formulação e avaliação de políticas públicas e programas, bem como para a reflexão sobre práticas acadêmico-empresariais visando a inovação e desenvolvimento de tecnologias.

Palavras-chave: Inovação. Tecnologia. Empresa de Base Tecnológica. Licenciamento.

\section{Development of technologies and businesses in a program of incentive to innovation}

\begin{abstract}
This research analyzes the development of technology in the academic environment and its consolidation in technological businesses in micro, meso and macro organizational levels. Based on the content analysis of reports regarding a governmental Brazilian innovation incentive program, the Programa de Incentivo à Inovação, the research highlights the diffused actions of researchers-entrepreneurs in the micro level, which oscillates around the undertaking and the licensing of technology when creating spinoffs. In the meso level, among clients, competitors, partners and suppliers, the academic spinoffs operate under tight knit rules of regulatory agencies, as well as a strict dependence of financial support from research agencies and governmental programs for their startup. In the macro level, the political, legal, economical, technological, sustainable and cultural dispositions of the environment are important references for innovation agents to act. The findings of this study contribute to the formulation and evaluation of public policies and programs, as well as academic-business practices aimed at innovation and development of technologies.
\end{abstract}

Keywords: Innovation. Technology. Academic Spinoff. Licensing. 


\section{INTRODUÇÃO}

Dentre as atividades exercidas pelas universidades, encontra-se a promoção da pesquisa científica. A pesquisa, quando alinhada com políticas científico-tecnológicas e articulada no espaço entre universidade e comunidade, possibilita a geração de inovações e a criação de negócios que potencializam o desenvolvimento socioeconômico do país. Embora não detenha a exclusividade das inovações, "[...] a Universidade ainda é o centro principal de produção do conhecimento em todo o mundo" (FAVA-DE-MORAES, 2000, p. 10).

No Brasil, embora não se tenham números tão precisos da inovação tecnológica de origem acadêmica, a partir de um conjunto de variáveis que tangencia ora a inovação ora os aspectos de ciência e tecnologia, é possível visualizar um panorama nacional: os programas de mestrado e doutorado, de 1996 a 2014, cresceram cerca de 200\%, sendo 420.283 o número de mestres e doutores no país (CGEE, 2016); os depósitos de pedidos de patentes pelas universidades e instituições de ensino, ao longo de quinze anos (1990-2004), subiu de cerca de 40 para mais de 200 por ano (INPI, 2007). Também foi notável que dentre os 50 principais depositantes do total de patentes (20042008), 16 eram universidades e instituições de ensino (INPI, 2011).

Nesse contexto, o presente artigo analisa o desenvolvimento de inovações tecnológicas em ambiente acadêmico e a consolidação dessas em negócios, notadamente sob a forma de licenciamentos para empresas estabelecidas ou por meio da criação de empresas de base tecnológica (EBTs). Os dados deste estudo foram compilados de relatórios do Programa de Incentivo à Inovação (PII), entre os anos de 2007 e 2010, e são referentes às experiências advindas de instituições públicas de ensino, pesquisa e extensão, quais sejam: Universidade Federal de Minas Gerais (UFMG), Universidade Federal de Lavras (UFLA) e Universidade Federal de Juiz de Fora (UFJF). O PIl é uma iniciativa da Secretaria de Estado de Ciência, Tecnologia e Ensino Superior de Minas Gerais (SECTES/MG), em parceria com o Serviço Brasileiro de Apoio às Micro e Pequenas Empresas (SEBRAE-MG), universidades federais e prefeituras locais, que, em conjunto, buscam articular e adensar competências, promovendo a inovação tecnológica no ambiente acadêmico.

A fundamentação teórica sobre a inovação tecnológica no ambiente acadêmico e sua trajetória para a viabilização de negócios tecnológicos será abordada na próxima seção. Na sequência, são relatados os procedimentos metodológicos adotados para o desenvolvimento deste estudo, baseados na análise de conteúdo e na elaboração de um quadro de referência sobre os dados coletados. A discussão dos resultados e análises evidenciam que as dinâmicas de desenvolvimento de tecnologias e de negócios tecnológicos são permeadas por uma rede de inovação composta por vários agentes, processos e recursos. Ademais, as considerações finais sumarizam os principais resultados do estudo, assim como são apresentados os seus limites e questões que poderão subsidiar o desenvolvimento de futuras pesquisas.

\section{INOVAÇÃO TECNOLÓGICA NO AMBIENTE ACADÊMICO}

A inovação tecnológica aplica-se tanto a produtos (bens e serviços) ou a processos, e pode ser entendida pela ocorrência de (a) melhoria significativa no desempenho de um produto; (b) redução de características indesejadas do produto ou processo; e (c) surgimento de novidades anteriormente inexistentes (HAGE; HOLLINGSWORTH, 2000).

É comum que a inovação tecnológica ocorra por meio de interfaces entre a universidade e o mercado, demandando competências que envolvem pesquisas, desenvolvimento de produtos/ processos e comercialização (CUNHA, 1999; HAGE; HOLLINGSWORTH, 2000). É desejável que as tecnologias desenvolvidas sejam protegidas por meio de patentes e outros registros (AMADEl; TORKOMIAN, 2009), tornando-se negócios principalmente por meio dos licenciamentos para empresas estabelecidas ou da criação de Empresas de Base Tecnológica de Origem Acadêmica (EBTs de OA). A patente é um título de propriedade industrial sobre a invenção ou modelo de utilidade e garante ao inventor certa segurança nas negociações com outras partes interessadas em comprar a tecnologia para aplicação com fins comerciais (LOBOSCO; MORAES; MACCARI, 2010). As demais formas existentes no contexto brasileiro são os registros de marca, de software ou de desenho industrial (INPI, 2007).

As EBTs de origem acadêmica, também conhecidas como spinoffs acadêmicos, são organizações criadas para explorar comercialmente as tecnologias desenvolvidas nos laboratórios universitários (ROBERTS, 1991; WRIGHT et al., 2008) ou ao processo de transformação dos resultados de pesquisas 
acadêmicas em tecnologia para o desenvolvimento de produtos e negócios (NDONZUAU; PIRNAY; SURLEMONT, 2002; O'SHEA; CHUGH; ALLEN, 2008). Para além do ambiente acadêmico, as EBTs surgem pelo licenciamento formal da tecnologia para os empreendedores interessados em comercializar os produtos oriundos das patentes, para empresas estabelecidas ou até mesmo para a empresa em que o pesquisador acadêmico participa da equipe de empreendedores (ROBERTS; MALONE, 1996; ROGERS; TAKEGAMI YIN, 2001). De forma ampla, essas EBTs são empreendimentos criados para explorar a propriedade intelectual desenvolvida nas instituições acadêmicas (SHANE, 2004; O'SHEA; CHUGH; ALLEN, 2008).

As EBTs representam uma importante forma de mobilização de recursos e de desenvolvimento sociotécnico para a própria universidade, e de grande interesse para a sociedade (FRANKLIN; WRIGHT; LOCKETT, 2001). Subjacentes à criação de uma EBT encontram-se diversos fatores, tais como as características individuais dos envolvidos, a configuração organizacional da empresa, os recursos necessários ao empreendimento, a ambiência externa e a relação com fornecedores, competidores e clientes, o processo de desenvolvimento do negócio e da tecnologia, além do contexto sociocultural mais amplo (O'SHEA; CHUGH; ALLEN, 2008; SILVA; REIS, 2015).

O suporte das universidades na promoção das tecnologias geradas pelos pesquisadores é fundamental para que essas possam chegar ao mercado com mais chance de sucesso (RASMUSSEN; $\mathrm{BORCH}, 2010)$. Comumente, esse suporte advém do apoio concedido pelos Escritórios de Transferência de Tecnologia (ETTs) ou Núcleos de Inovação Tecnológica (NITs). Como órgãos internos às universidades, os ETTs são especializados em licenciamento e transferência de tecnologias para empresas estabelecidas ou empresas nascentes (SIEGEL et al., 2004). Eles auxiliam os pesquisadores na elaboração de solicitação de patentes, nas negociações, bem como na elaboração de contratos e convênios com as organizações de mercado. Os ETTs também podem garantir o aporte de condições à criação de novas EBTs por meio de incubadoras de empresas e parques tecnológicos (CONDE; SANTOS, 1999).

A transferência da tecnologia remete à posse e ao direito da patente, enquanto que o licenciamento aufere o direito de explorar comercialmente a tecnologia mediante o pagamento de royalties pelo licenciado ao titular da patente. O licenciamento é um processo que consiste de várias etapas, como pesquisa científica, descoberta, proteção e divulgação da tecnologia para o mercado, negociações e formalização de contrato (SIEGEL et al., 2004).

As políticas de fomento à inovação possuem presença significativa no ambiente acadêmico. Parte dos recursos destinados aos investimentos em Pesquisa, Desenvolvimento e Inovação (PD\&I) é comumente oriunda de fontes de recursos governamentais, demonstrando a importância do setor público e das políticas públicas no incentivo à inovação (GALLON; REINA; ENSSLIN, 2010).

Embora seja crucial a habilidade das universidades em gerar de forma bem-sucedida as EBTs, diversas restrições e regulamentações internas podem afetar esse objetivo. As limitações são manifestas, por exemplo, na falta de um entendimento sobre as leis que impactam na inovação, em incentivos tímidos para que os pesquisadores gerem novos empreendimentos, ou até mesmo em conflitos desses próprios pesquisadores perante o desafio do processo empreendedor e da carreira acadêmica (ROBERTS, 1991).

Como observado, o desenvolvimento de tecnologias e a geração de negócios tecnológicos em ambiente acadêmico guarda sua complexidade, sendo que a compreensão de suas nuances representa fator distintivo não só para o desenvolvimento de tecnologias e empreendimentos bemsucedidos, como também para a avaliação das políticas científico-tecnológicas e para a articulação entre universidades, pesquisadores e mercados.

\section{CONTEXTO INVESTIGADO E MÉTODOS DE PESQUISA}

O Programa de Incentivo à Inovação (PII) promove a inovação tecnológica, proteções e transferências de tecnologia tanto por licenciamentos quanto pela abertura de novas empresas (PROGAMA..., 2009). Entre os anos de 2007 (início do programa) e 2010, foram implementados 8 Plls em Universidades Federais, a saber: 2 na UFLA (Lavras), 1 na UNIFEl (Itajubá), 2 na UFJF (Juiz de Fora), 1 na UFV (Viçosa), 1 na UFMG e outro na Biominas (Minas Gerais). O PII de Lavras foi o primeiro a ser realizado (primeira rodada) e os seus resultados forneceram melhorias para a implementação do PII UNIFEl e UFJF (segunda rodada). Por sua vez, estes contribuíram para melhorias no Plls subsequentes (UFV, UFMG e Biominas - terceira rodada). Assim, foram selecionados para análise os relatórios do PII UFLA (PROGRAMA..., 2008), UFJF (PROGRAMA..., 2009) e UFMG (PROGRAMA..., 2010), por abordarem 
a primeira, a segunda e a terceira rodada respectivamente, propiciando uma representação da evolução do programa ao longo do tempo.

O PII é dividido em duas fases: a primeira, direcionada para a elaboração dos Estudos de Viabilidade Técnica, Econômica, Comercial, Impacto Ambiental e Social (EVTECIAS); a segunda, direcionada para o desenvolvimento de protótipos e do plano tecnológico. Cada PIl contemplou 20 projetos na primeira fase, totalizando 60 projetos. A segunda fase totalizou 37 projetos (13 UFLA, 14 UFJF e 10 UFMG). Desse total, para fins deste estudo, foram analisados 24 projetos, conforme apresentado no Quadro 1.

Quadro 1 - Caracterização dos Plls e Projetos Analisados

\begin{tabular}{|c|c|c|c|c|c|}
\hline $\begin{array}{l}\text { Instituição } \\
\text { Período }\end{array}$ & $\begin{array}{l}\text { Projetos } \\
\text { Analisados }\end{array}$ & Detalhe dos Projetos & $\begin{array}{l}\stackrel{0}{\frac{3}{2}} \\
\frac{0}{0} \\
\text { 은 }\end{array}$ & 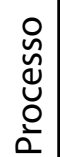 & 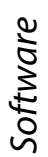 \\
\hline \multirow{9}{*}{$\begin{array}{l}\text { UFLA } 2007 \text { a } \\
2008\end{array}$} & Nematoides & Nematoides para controle biológico de pragas. & - & & \\
\hline & Alface & $\begin{array}{l}\text { Alface de folhas lisas, resistente a nematoides e tolerante } \\
\text { ao calor. }\end{array}$ & & • & \\
\hline & Leveduras & $\begin{array}{l}\text { Leveduras selecionadas para a produção de cachaça de } \\
\text { qualidade. }\end{array}$ & & • & \\
\hline & $\begin{array}{l}\text { Bioativador do } \\
\text { Solo }\end{array}$ & $\begin{array}{l}\text { Blend com uma concentração específica entre o } \\
\text { Lithothamnium e o Biotech com alto valor agregado e } \\
\text { com função ativadora. }\end{array}$ & • & & \\
\hline & Sêmen & $\begin{array}{l}\text { Bio-speckle laser como quantificador de atividade } \\
\text { biológica em sêmen animal. }\end{array}$ & • & & \\
\hline & $\begin{array}{l}\text { Sementes } \\
\text { Híbridas de Milho }\end{array}$ & Produção de sementes híbridas de milho. & & - & \\
\hline & Couro & Recuperação do cromo. & & - & \\
\hline & $\begin{array}{l}\text { Propagação Veget. } \\
\text { de Cafeeiros }\end{array}$ & $\begin{array}{l}\text { Protocolo para propagação vegetativa de cafeeiros } \\
\text { (Coffea arábica L.) por meio de estacas caulinares. }\end{array}$ & & • & \\
\hline & $\begin{array}{l}\text { Bioprotetor do } \\
\text { Café }\end{array}$ & $\begin{array}{l}\text { Aplicação de um agente como bioprotetor de café e de } \\
\text { seus metabólicos na indústria de alimentos. }\end{array}$ & $\bullet$ & & \\
\hline \multirow{8}{*}{$\begin{array}{l}\text { UFJF } 2007 \text { a } \\
2009\end{array}$} & Handgrip & Avaliação da força de preensão da mão. & - & & - \\
\hline & Kit Estéril & $\begin{array}{l}\text { Kit estéril e descartável para a realização da estereotaxia } \\
\text { (procedimento radiográfico para marcação de lesões } \\
\text { mamárias). }\end{array}$ & $\bullet$ & & \\
\hline & $\begin{array}{l}\text { Fluxômetro de } \\
\text { Urina }\end{array}$ & $\begin{array}{l}\text { Fluxômetro portátil que utiliza a variação da capacidade } \\
\text { elétrica para determinar o volume da urina. }\end{array}$ & • & & • \\
\hline & Água no Leite & $\begin{array}{l}\text { Verificação da porcentagem de água no leite por meio da } \\
\text { medição da condutividade elétrica. }\end{array}$ & - & & \\
\hline & Tacômetro & $\begin{array}{l}\text { Equipamento para a medição do nível de balanceamento } \\
\text { de máquinas rotativas. }\end{array}$ & - & & \\
\hline & Reator & $\begin{array}{l}\text { Reator eletrônico de baixo custo que propicia correntes } \\
\text { elétricas em alta frequência, a partir da rede elétrica de } \\
\text { baixa frequência. }\end{array}$ & • & & \\
\hline & Pavimentos & $\begin{array}{l}\text { Processo para a detecção de defeitos em pavimentos } \\
\text { asfálticos com a utilização de imagens. }\end{array}$ & • & & - \\
\hline & Modem PLC & $\begin{array}{l}\text { Dispositivo de transmissão digital - voz, dados e vídeo - } \\
\text { através das redes de distribuição de energia elétrica: PLC }\end{array}$ & • & & \\
\hline
\end{tabular}




\begin{tabular}{|c|c|c|c|c|}
\hline \multirow{5}{*}{$\begin{array}{l}\text { UFMG } 2009 \\
\text { a } 2010\end{array}$} & Órtese & $\begin{array}{l}\text { Próteses/órteses são aparelhos que auxiliam as funções } \\
\text { prejudicadas de membros de indivíduos portadores de } \\
\text { deficiência física. }\end{array}$ & $\bullet$ & \\
\hline & Vidros Bioativos & $\begin{array}{l}\text { Materiais capazes de se ligar ao tecido ósseo propiciando } \\
\text { a mineralização do tecido e sua regeneração. }\end{array}$ & $\bullet$ & \\
\hline & Sensor & $\begin{array}{l}\text { Tecnologia (Chip) de Redes de Sensores dedicados sem } \\
\text { Fio (RSSF). }\end{array}$ & - & \\
\hline & Monitoramento & $\begin{array}{l}\text { Sistema de controle de cargas elétricas de baixo custo e } \\
\text { fácil instalação. }\end{array}$ & $\bullet$ & $\bullet$ \\
\hline & $\begin{array}{l}\text { Prontuário } \\
\text { Eletrônico }\end{array}$ & $\begin{array}{l}\text { Software de Registro Eletrônico em Saúde (RES) } \\
\text { que possui um conjunto de módulos com várias } \\
\text { funcionalidades. }\end{array}$ & & $\bullet$ \\
\hline
\end{tabular}

Fonte: Elaborado pelos autores a partir de Programa... $(2008,2009,2010)$.

Os relatórios do Pll refletem as percepções de pesquisadores, empreendedores e agentes de suporte à inovação. Seus dados são oriundos de fontes primárias, coletadas por meio das entrevistas semiestruturadas com os pesquisadores, assim como de fontes secundárias, tais como relatórios de pesquisas. Um aspecto que impactou na seleção de conteúdo dos relatórios analisados foi o consentimento dos pesquisadores para análise e publicação dos dados dos projetos.

O material selecionado foi submetido à análise de conteúdo para identificar categorias e conceitos centrais para a compreensão das dinâmicas de inovação, que nesse caso são potencializadas por uma política pública. Para Vergara (2005, p.15), essa é uma técnica "para o tratamento de dados que visa identificar o que está sendo dito a respeito de determinado tema", que neste artigo refere-se às dinâmicas subjacentes ao desenvolvimento de tecnologias e aos negócios tecnológicos nos projetos do PII-MG.

A análise de conteúdo compreende três etapas básicas: (a) pré-análise; (b) exploração do material; e (c) tratamento dos dados e interpretação. A pré-análise refere-se à seleção do material propriamente dito; no caso dos relatórios do Pll, estes foram obtidos junto ao NTQI/UFMG. A exploração do material busca alinhar o conteúdo do texto com os objetivos da pesquisa. Bardin (1977) salienta que o conteúdo é analisado de forma a substituir a leitura "normal" - no caso aquela inicialmente realizada nos relatórios - pela leitura do analista, que é atraído pelo "[...] potencial de inédito (do não-dito), retido por qualquer mensagem" (BARDIN, 1977, p. 9).

Foram selecionados e explorados os conteúdos alinhados com o objetivo do artigo, sendo que os relatórios do PIl são mais amplos e abordam informações diversas. O tratamento dos dados e a interpretação dos mesmos ocorrem por meio da geração iterativa de conceitos e categorias de análise que, por sua vez, são submetidos a um processo seletivo e relacional, visando-se a elaboração de descrições ou de teorias sobre o tema em questão (BARDIN, 1977).

No caso deste estudo, conceitos e categorias foram analisados, tendo a relação entre os mesmos sido esquematizada no diagrama analítico apresentado na próxima seção do artigo (Figura 1). Em relação às informações contidas na figura, observa-se que nem todos os conceitos, categorias e relações estabelecidas entre os elementos dos vários níveis se aplicam simultaneamente ao contexto individual de cada projeto analisado.

Salienta-se que, para a análise de conteúdo e para a elaboração do diagrama analítico foram empregados os softwares NVivo e CmapTools.

\section{DO DESENVOLVIMENTO DE TECNOLOGIAS AOS NEGÓCIOS TECNOLÓGICOS NOS PROJETOS DO PROGRAMA DE INCENTIVO À INOVAÇÃO (PII-MG)}

O desenvolvimento de tecnologias em ambiente acadêmico e sua consolidação em negócios, seja por meio da criação de empresas de base tecnológica (EBTs), seja como licenciamentos para empresas estabelecidas, envolve uma rede de inovação composta por vários agentes, processos e recursos, cujas configurações diversas contribuem para o sucesso ou fracasso dos negócios. Baseando-se no modelo de Djokovic e Souitaris (2008), a Figura 1 agrupa esses elementos em três níveis de análise - micro, meso e macro. 
Figura 1 - Desenvolvimento de Tecnologias e Negócios Tecnológicos nos Projetos do PII

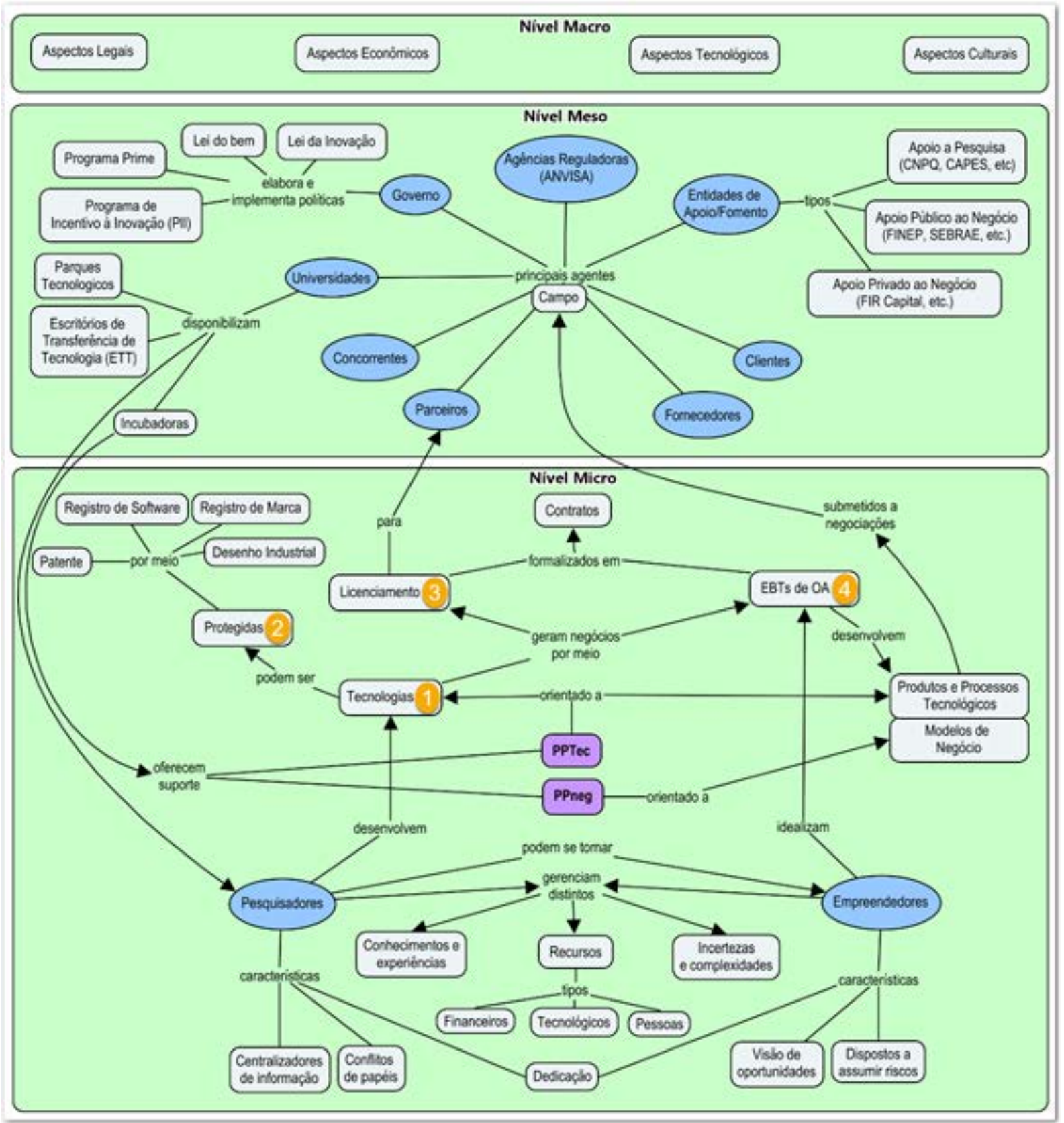

Fonte: Elaborado pelos autores (2016).

\subsection{Nível micro}

O nível micro compreende os processos, recursos, pessoas e informações que são articulados, inicialmente, para o suporte e o desenvolvimento de tecnologias nos ambientes acadêmicos, como indicado pela marcação (1) na Figura 1. Engloba pesquisadores que mobilizam conhecimentos, experiências e recursos diversos, num cenário de incertezas e complexidades, onde a tecnologia pode nem mesmo chegar a gerar negócios. As tecnologias pertencem às diversas áreas de conhecimento, tais como ciências biológicas, ciências exatas, dentre outras. Ainda, as tecnologias remetem a mercados específicos, como agronegócio, energia, transporte, indústria, comércio etc. Os projetos do Pll, em sua maioria, envolvem tecnologias de produtos e processos e, em alguns casos, o desenvolvimento de softwares.

Quando gerada, a tecnologia é submetida a alguma forma de proteção (2). A preocupação com a proteção da tecnologia pode ser constatada no Proj. Bioprotetor, no qual os pesquisadores mostraram-se reservados em disponibilizar para a equipe do Pll as informações referentes à tecnologia, visto que a mesma passava pelo processo de proteção intelectual no Instituto Nacional 
da Propriedade Industrial (INPI).

Protegidas, as tecnologias podem ser licenciadas para outras empresas estabelecidas no mercado (3) ou para empresas nascentes - EBTs (4). Os processos desde o desenvolvimento da tecnologia ao estabelecimento do negócio apresentam peculiaridades em cada projeto. Em casos como o do Proj. Kit Estéril, o desenvolvimento da tecnologia se deu ao longo da tese de doutorado do pesquisador e culminou, logo em seguida, em um licenciamento. No Proj. Handgrip, observase um período de cerca de 20 anos entre o desenvolvimento do primeiro protótipo da tecnologia, iniciando na dissertação de mestrado do pesquisador (1989), passando por sua tese de doutorado e por desdobramentos posteriores, gerando, por fim, uma EBT (SensorMed).

Para aumentar as chances de sucesso na concepção da tecnologia, os pesquisadores são assessorados pelo Processo de Planejamento Tecnológico (PPTec) auxiliando a integração da tecnologia, produto e mercado com vista ao protótipo comercial. Durante as diversas fases do PPTec, os pesquisadores gerenciam pessoas e recursos como máquinas, equipamentos e insumos necessários para a pesquisa.

Salienta-se que nem sempre a constituição de uma EBT é a melhor opção de negócio, sendo comum a prática de licenciamento da tecnologia para empresas estabelecidas no mercado. Caso a opção seja o licenciamento, é necessário identificar e estabelecer negociações com possíveis empresas interessadas. $O$ processo mais comum envolve a licitação, em que o escritório de transferência da universidade (ETT) se responsabiliza tanto pelas negociações quanto pela formalização dos acordos. Não obstante, pode ocorrer de os pesquisadores fazerem uso de suas próprias redes de relacionamento para encontrar as empresas mais adequadas para estabelecerem contratos de licenciamento. No Proj. Alface, o pesquisador iniciou esse processo com a empresa SAKATA, já estabelecida no mercado, e com a qual tinha um contato próximo. A relação de confiança previamente existente entre as partes fornecia certa garantia ao pesquisador, visto que sua tecnologia não poderia ser patenteada, necessitando apenas de registro no Ministério da Agricultura, Pecuária e Abastecimento (MAPA). No Proj. Leveduras, a transferência alcança dimensões mais complexas e distantes do pesquisador, dado que apenas empresas canadenses e chinesas dispunham do maquinário necessário para aplicação da tecnologia; no Brasil, foram identificadas duas empresas (LNF Latino Americana e Fermentec) que subcontratavam as empresas canadenses e chinesas, sendo que uma delas se mostrou interessada em obter a tecnologia.

Já a criação de uma EBT (4) coloca o pesquisador em posição de empreendedor. Os pesquisadoresempreendedores, em conjunto com equipes de suporte das universidades e de outros órgãos de apoio, necessitam gerenciar os recursos financeiros, tecnológicos ou pessoas, articulando-os com seus conhecimentos e experiências, num cenário de incertezas e complexidades bastante distinto daquele que envolvia somente o desenvolvimento da tecnologia. Observa-se que muitas das equipes em configurações ideais para o desenvolvimento da tecnologia, mostram-se insuficientes (em termos qualitativos e quantitativos) para levar a cabo um empreendimento. Restrições objetivas se apresentam: nem sempre estes professores e suas equipes são conhecedores de princípios, técnicas e ferramentas gerenciais capazes de potencializar e de confluir o seu conhecimento em uma ação concreta, prática e útil à finalidade de comercialização da inovação.

As redes de relacionamento do pesquisador estão geralmente voltadas para o ambiente acadêmico, necessitando de direcionamento para o mercado. Nesse sentido, para aumentar as chances de uma tecnologia gerar uma EBT, os pesquisadores-empreendedores são assessorados não só pelo PPTec (vide observações anteriores), como também pelo Processo de Planejamento do Negócio (PPNeg). O PPNeg possibilita o desenvolvimento dos processos organizacionais que comporão o negócio tecnológico, com vistas à configuração de um produto e à definição de um modelo de negócio adequado para a exploração comercial da tecnologia desenvolvida nos laboratórios das universidades. Nesse processo também está incluído o Estudo de Viabilidade denominado EVTECIAS, que explicita os principais dados que serão requisitados para a posterior avaliação dos projetos pelos potenciais investidores, futuros parceiros e agências de patentes.

Alguns projetos que originam EBTs podem comercializar suas tecnologias por meio dos produtos ou até mesmo de serviços como é o caso do Proj. Monitoramento e Proj. Pavimentos. Dentre algumas das EBTs criadas a partir de projetos do PIl, menciona-se a SensorMed (originado do Proj. Handgrip), responsável pela montagem dos equipamentos (dinamômetro manual, capaz de medir a força do indivíduo) e sua distribuição no mercado. No Proj. Bioprotetor do Café, empreender significou construir uma biofábrica para a produção dos agentes biológicos, que são biocontroladores de fungos prejudiciais à qualidade do café. Já no Proj. Sêmen, inicialmente pretendeu-se comercializar um equipamento para centrais de certificação de sêmen bovino. Todavia, a configuração final do 
negócio foi modificada de produto para serviço, a partir da criação de laboratório que realiza os testes de certificação de sêmen para as centrais.

A dinâmica entre licenciamento ou criação de EBTs, por vezes, ultrapassa a dimensão técnica e processual do negócio. Constata-se a dúvida de alguns pesquisadores em empreender ou licenciar a tecnologia. Surgem também conflitos de papéis, onde os pesquisadores, embora pretendam empreender, não querem ser vistos como empresários. Observa-se também que os pesquisadores apresentam uma tendência de centralizar as informações das tecnologias, que para além de uma possível característica pessoal, faz parte do sigilo que precede a proteção da tecnologia. Em alguns casos, essa centralização leva à interrupção de projetos, como ocorrido com um pesquisador que assumiu um cargo no CNPq e não nomeou ou indicou alguém para dar continuidade às atividades.

É sugestivo que algumas características sejam comuns aos grupos que lidam com o licenciamento ou com a criação de EBTs: ambos lidam com incertezas e complexidades, e a dedicação à ideia (inovação tecnológica) representa fator distintivo para o sucesso subsequente. Os dois grupos também lidam com a gestão de recursos diversos, articulados em torno de seus conhecimento e experiência. Todavia, observa-se considerável distância entre experiência/conhecimento acadêmico e aquele demandado pela ação empreendedora. Ademais, observa-se que a amálgama pesquisadorempreendedor só se completa quando os indivíduos possuem características intrínsecas como a visão de oportunidades e a disposição para assumir riscos.

Como observado, cada um dos projetos do PII apresenta especificidades que ora propulsionam, ora restringem o desenvolvimento de inovações e negócios tecnológicos. A Tabela 1 apresenta uma síntese dos resultados alcançados nos projetos analisados neste estudo, considerando-se os projetos com pedidos de patente, projetos que originaram EBTs e projetos que realizaram licenciamentos. Também é apresentada a proporção desses resultados em relação ao total de projetos do PII (60 projetos na primeira etapa e 37 na segunda), como um complemento à análise qualitativa que aborda 24 projetos desse universo. Observa-se a importância do programa para o incentivo à proteção intelectual e um equilíbrio entre o número de empresas criadas (13) e o número de licenciamentos realizados (10) no universo de 60 projetos apurados.

Tabela 1 - Síntese dos Resultados do PII (2007-2010)

$\begin{array}{lcccccc} & \text { UFLA } & \text { UFJF } & \text { UFMG } & \text { Total } & \begin{array}{c}\text { \% sobre a 1a } \\ \text { etapa } \\ \text { 60 projetos }\end{array} & \begin{array}{c}\% \text { sobre a 2a } \\ \text { etapa }\end{array} \\ \text { 37 projetos }\end{array}$

Fonte: Elaborado pelos autores (2016).

Embora os elementos do nível micro possibilitem as inovações tecnológicas e o caminho até que sejam licenciadas ou direcionadas para a criação de EBTs, observam-se interações com outros elementos posicionados nos demais níveis (meso/macro) da ambiência, os quais serão explorados a seguir.

\subsection{Nível meso}

O nível meso envolve os agentes que compõem o campo organizacional e que desempenham papéis distintos, relacionados com atividades que vão do suporte à inovação até a criação de uma EBT propriamente dita. A dinâmica de operação desse nível alinha-se com as configurações dos elementos do nível micro e com as disposições encontradas no nível macro. De modo geral, são considerados como principais agentes da inovação no nível meso: clientes, concorrentes, parceiros, fornecedores, entidades de apoio e fomento, governo, agências reguladoras e universidades. Em função das distintas tecnologias, esses agentes variam bastante, conforme ilustram alguns dos projetos do PII:

a) Clientes: no Proj. Nematoides os clientes são os produtores de alimentos orgânicos; no Proj. Sêmen, as centrais certificadoras de sêmen bovino, envolvidas na cadeia de produção e distribuição de sêmen, materiais e equipamentos de uso na inseminação artificial e de outros produtos ligados à reprodução animal. O Proj. Handigrip apresentou uma segmentação de clientes bastante variada, 
incluindo hospitais, clínicas, unidades de exames de habilitação, fisioterapeutas, ortopedistas, educadores físicos, além de academias, clubes, times; e canais diretos entre empresas (business to business), neste caso, os fabricantes de esteiras ergométricas e bicicletas. Os potenciais clientes do Proj. Kit Estéril atuam na área médica, como os hospitais e as clínicas de medicina nuclear e radiologia, sendo que a tecnologia do kit se apresentou como alternativa avançada e confiável para o diagnóstico de câncer de mama.

b) Concorrentes: se por um lado o Proj. Handgrip apresenta um alto potencial de clientes, por outro, constata-se que existe um grande número de empresas que comercializam produtos similares, configurando um cenário que se aproxima de uma "concorrência perfeita". No Proj. Sementes Híbridas de Milho, a concorrência é mais específica: as empresas Monsanto, Pionner, Agroceres, Syngenta e Embrapa. Alguns projetos, por serem muito inovadores, apresentam somente concorrentes indiretos por meio de produtos substitutos, como é o caso do Proj. Kit Estéril, que inclusive conquistou o 10 lugar no Austin Technology Incubator Challenge.

c) Parceiros: para o desenvolvimento de sua tecnologia, o Proj. Handgrip necessitou estabelecer parceria com a empresa EMG System - Eletromiografia e Biofeedback - sediada inclusive em outro Estado (SP).Considera-se olicenciamento da tecnologia como uma espécie de parceria, como ocorrido entre o Proj. Alface e a empresa SAKATA. A empresa Wisecomm do Proj. Health Care firmou a parceria com a empresa GeoTech, para o desenvolvimento de produtos para uma plataforma de continuidade do cuidado; e com a empresa Axoon para o desenvolvimento do chip para equipamentos médicos.

d) Fornecedores: o Proj. Handgrip necessita de fornecedores para a fabricação de empunhaduras e sensores que compõem seu equipamento final. No Proj. Sementes Híbridas de Milho, o fornecedor assume a terceirização de parte do processo de beneficiamento de sementes, já que não se dispõe de todo o equipamento necessário para a implementação da tecnologia. Em outros projetos, os produtos dos fornecedores são comercializados simultaneamente com a tecnologia em questão, como é o caso do Proj. Prontuário, em que o software desenvolvido só é entregue para o cliente juntamente com a hospedagem em um servidor web (responsável pelo armazenamento de páginas do software) do fornecedor do projeto.

e) Agências reguladoras: testes e registros que garantam a eficiência dos produtos ou o controle dos impactos destes sobre o homem e o meio ambiente são mais comuns para produtos da área química, farmacológica e biológica. Para que a empresa oriunda do Proj. Vidros Bioativos entrasse efetivamente em funcionamento, foi necessária a Autorização de Funcionamento de Empresa (AFE), obtenção do Certificado de Boas Práticas de Fabricação (CBPF), laudo de conformidade do produto, concedidos pela Agência Nacional de Vigilância Sanitária (ANVISA). Situação análoga ocorreu com o Proj. Sementes Híbridas de Milho, que necessitou de registro junto ao Ministério da Agricultura, Pecuária e Abastecimento (MAPA).

f) Entidades de apoio/fomento: comumente, o apoio/fomento ocorre por meio da disponibilização de recursos públicos ou privados para a aquisição de insumos, equipamentos, bolsas de pesquisas, entre outros. O desenvolvimento de tecnologias geralmente conta com o apoio/fomento de entidades públicas como o Conselho Nacional de Desenvolvimento Científico e Tecnológico (CNPQ), a Coordenação de Aperfeiçoamento de Pessoal de Nível Superior (CAPES) e as Fundações Estaduais de Amparo à Pesquisa. Dentre as públicas, mencionam-se a Financiadora de Estudos e Projetos (FINEP), as Secretarias Estaduais de Ciência e Tecnologia (no caso do PII, a SECTES$M G$ ) e os bancos de desenvolvimento (BNDES, BDMG, outros). Dentre as entidades privadas, existem diferentes tipos de investidores conforme os níveis de desenvolvimento do negócio, como os angels e fundos de capital semente para as empresas que se encontram nos estágios iniciais, além dos privaty equities, quando a empresa já está mais consolidada, a exemplo da FIR Capital (venture capital). Além desses investidores, existem empresas privadas com interesse no desenvolvimento de novas tecnologias para a melhoria de seu portfólio de produtos ou de seus processos industriais. O Proj. Modem PLC, por exemplo, recebeu um significativo investimento da CEMIG para o desenvolvimento da tecnologia, que consiste em um dispositivo de comunicação digital de transmissão de dados multimídia por meio das redes de distribuição de energia elétrica.

g) Governo: elabora e implementa, em suas diversas instâncias, as políticas e os programas de incentivo e apoio à inovação, a exemplo do Pll. A extensão e a importância deste agente se desdobra em contextos outros, como aqueles relacionados com as agências reguladoras, entidades de apoio/fomento e as próprias universidades nas quais as tecnologias são geradas. Outro programa governamental de âmbito nacional é o Primeira Empresa Inovadora (PRIME), coordenado pela FINEP que oferece apoio a empresas com até dois anos de vida, tendo em vista o desenvolvimento e inserção no mercado de produtos inovadores. Também está no escopo da atuação governamental a 
criação de incentivos, redução ou isenção de impostos para empresas que desenvolvam produtos e serviços tecnológicos, dentre outros.

h) Universidades: comportam o desenvolvimento das pesquisas e das tecnologias, fornecendo infraestrutura laboratorial e insumos. Possuem, em certos casos, estruturas específicas de suporte à inovação, como os parques tecnológicos, as incubadoras e os escritórios de transferência de tecnologia (encarregados da proteção intelectual e de negociações das tecnologias com o mercado). No conjunto, esses ativos promovem um ambiente favorável ao compartilhamento de recursos e conhecimentos, aumentando as possibilidades de êxito dos negócios acadêmicos. Todos os relatórios do PIl evidenciam algum grau de envolvimento das pró-reitorias de pesquisa, dos ETTs ou das incubadoras nos resultados de inovação. Não obstante toda essa estrutura, os pesquisadores constituem os agentes de inicialização do processo de geração de tecnologias.

\subsection{Nível macro}

O nível macro abrange os aspectos mais estruturais que interferem nas dinâmicas organizacionais e, em última instância, na forma de pensar e agir dos indivíduos. Esse nível, tal como considerado no quadro analítico desenvolvido neste estudo, envolve os aspectos legais, econômicos, tecnológicos e culturais do ambiente.

a) Aspectos legais: no contexto da inovação, a Lei do Bem (no 11.196/ 2005) consolidou os incentivos fiscais que as pessoas jurídicas podem usufruir desde que realizem pesquisa tecnológica e desenvolvimento de inovação tecnológica. A Lei da Inovação, no 10.973/2004 (BRASIL, 2004) estabeleceu medidas de incentivo à pesquisa e à inovação, além de ter determinado os mecanismos de gestão para as instituições científicas e tecnológicas. Quando uma tecnologia é gerada, uma primeira interação com os aspectos legais se dá diante da possibilidade de protegê-la conforme prevê a Lei no 9.279/1996 (BRASIL, 1996), que regula a propriedade industrial. O licenciamento da tecnologia para terceiros é mediado também pela Lei da Inovação (BRASIL, 2004). Já a criação de uma EBT demandará a constituição jurídica de uma empresa, em conformidade com a Lei 10.406/2002 Código Civil que regula a constituição de pessoas jurídicas (BRASIL, 2002). Além dessas demandas, a EBT necessitará obter outros registros em diversos órgãos para poder comercializar a tecnologia. $\mathrm{O}$ Proj. Alface, por exemplo, optou por licenciar o direito comercial de explorar a tecnologia desenvolvida. De forma distinta, no Proj. Handgrip, a opção foi pela criação da empresa SensorMed. A SensorMed necessitou obter o registro do CNPJ, inscrição estadual, certificação ANVISA do produto, além do registro no INPI da patente e marca "Handgrip", só para citar as principais interações com os aspectos legais envolvidos na criação de uma EBT.

b) Aspectos econômicos: de uma maneira geral, todos os projetos passam por uma análise microeconômica, onde se busca identificar o potencial retorno a ser obtido em dado horizonte de tempo. Os projetos do PII de Lavras, com tecnologias voltadas para a agricultura, possuíam grande oscilação de demanda e preço, ora decorrente da sazonalidade do cultivo (Proj. Sementes Híbridas de Milho), ora das características de produtos relacionados à exportação e ao capital especulativo como cultura do café (Proj. Bioprotetor de Café e o Proj. Propagação Vegetativa de Cafeeiros), ou ainda devido a algumas tecnologias substituírem produtos importados, distribuídos por grandes multinacionais (Proj. Bioativador do Solo e Proj. Nematoides).

c) Aspectos tecnológicos: os projetos do Pll envolvem a pesquisa acadêmica de desenvolvimento tecnológico, como caracterizado no Quadro 1. Todavia, nem sempre todo o aparato tecnológico necessário para o suporte à inovação está disponível na universidade, principalmente quando da comercialização dos produtos. A exemplo do Proj. Sementes Híbridas de Milho, não se dispunha de equipamento próprio de beneficiamento de sementes, tendo sido necessário terceirizar parte do processo de produção em uma empresa pública, aproveitando a parceria já existente desta com a universidade. No caso do Proj. Couro, não se conhecia no mercado nenhum equipamento capaz de implementar em larga escala a tecnologia desenvolvida, configurando um gargalo tecnológico que demandou a identificação de empresas com competência técnica para desenvolver tal equipamento.

d) Aspectos culturais: os conhecimentos e valores da sociedade afetam de diferentes maneiras as tecnologias desenvolvidas. O Proj. Nematóides enfrentou resistência de clientes, que associavam os nematoides às pragas, além do fato do mercado de produtos orgânicos ser ainda incipiente e restrito devido ao preço mais elevado em comparação aos produtos convencionais. O Proj. Sêmen necessitou considerar que no contexto de inseminação artificial bovina, aspectos culturais ligados à certificação de sêmen ("maquiagem" do sêmen) remetem à importância da opinião das centrais de certificação sobre qualidade do sêmen, impossibilitando a comercialização direta para os criadores. Nessa 
direção, os aspectos culturais podem interferir, inclusive, na probabilidade do pesquisador se tornar um empreendedor, ressaltando a importância de estimular ainda mais a cultura empreendedora dentro das universidades.

\section{CONCLUSÃO}

As investigações que tratam exclusivamente da inovação no contexto brasileiro e com foco no ambiente acadêmico são mais recentes, mas já sinalizam um campo promissor investigação (CHENG; DRUMMOND; MATTOS, 2004; GONÇALVES; PAIVA JR., 2006; LEONEL et al., 2006; PEREIRA; MUNIZ, 2006; TONELLI; OLIVEIRA, 2007; REIS et al., 2006, 2007; SERRA et al., 2008; SILVA; REIS, 2015).

Os relatórios do Programa de Incentivo à Inovação (PII) constituem-se na principal base empírica deste artigo. Como mencionado, foram selecionados deste material apenas os conteúdos alinhados com a temática em estudo, constituindo-se, assim, em relevante fonte de informação secundária. Nesse sentido, tem-se a expectativa de que o presente estudo incentive outros pesquisadores a explorarem as possibilidades de análise de dados secundários, somando esforços com outras investigações que também fizeram uso desse artifício no contexto da inovação tecnológica (PACAGNELLA; PORTO; KANNEBLEY, 2006; RUTHES et al., 2006).

Não obstante, há que se considerar que os dados secundários geram certas restrições por não conterem todos os dados de que o pesquisador poderia dispor. Nesse sentido, sugere-se que novos estudos sejam desenvolvidos a partir da coleta de dados primários, sejam eles qualitativos ou quantitativos, de modo a ampliar a compreensão acerca das dinâmicas de desenvolvimento de tecnologias no ambiente acadêmico e a consubstanciação dessas em negócios tecnológicos.

Ainda visando o desenvolvimento de novos estudos, as teorias da agência (JENSEN; MECKLING, 1976) e da economia dos custos de transação (WILLIAMSON, 2002) elucidam potenciais agendas de pesquisa. A título sugestivo, registram-se algumas possibilidades exploratórias:

Pela teoria da agência, a organização é vista pelo conjunto de contratos que integram complexas relações e objetivos internos e externos conflitantes. A relação de agência propriamente dita constitui-se em um contrato no qual um "principal" contrata um "agente" para executar um serviço em seu nome, delegando responsabilidades e tomadas de decisão. Sobre as questões de licenciamento da tecnologia apresentadas neste artigo, o principal poderia ser representado pela universidade, enquanto os agentes, pelos pesquisadores que desenvolvem as tecnologias e por aqueles que optam por constituir uma EBT, além das empresas estabelecidas no mercado, interessadas em explorar comercialmente a tecnologia. Uma das formas de minimizar incertezas e riscos nas negociações é a proteção da tecnologia por meio de patente, registro de marca, registro de software ou desenho industrial.

Já pela teoria da economia dos custos de transação, independente dos jogos de mercado, uma grande quantidade de transações pode ocorrer dentro da organização. Assim, observam-se modos alternativos de coordenação: a organização (ou hierarquia) e o mercado. Em função do grau de especificidade de cada tipo de ativo, quanto mais específico esse ativo, maiores os custos de transação numa coordenação por meio do mercado. Nesse caso, a integração na hierarquia ou uma forma híbrida podem reduzir os custos de transação. Os projetos do Pll envolvem inovações tecnológicas (em produtos, processos e serviços), as quais podem ser consideradas ativos específicos, visto terem sido desenvolvidas/produzidas a partir de conhecimentos advindos de pesquisas especializadas. Essa especificidade do ativo é, inclusive, protegida na forma de patentes e outros tipos de registros de propriedade intelectual. Destarte, caso os custos de transação relacionados às inovações tecnológicas possibilitem sua produção e distribuição por meio da hierarquia, ocorre a constituição de uma EBT; casos os custos sejam impeditivos, busca-se por meio de transações com o mercado licenciar a tecnologia para alguma empresa já estabelecida. Ainda assim, a decisão de licenciar a tecnologia implica em outros custos de transação, que remetem aos mecanismos de monitoramento que serão necessários para garantir que o ativo não perca valor substancialmente, diante de uma apropriação indevida por parte da empresa licenciada.

Por fim, espera-se que o estudo desenvolvido contribua para a compreensão do desenvolvimento de tecnologias e de negócios tecnológicos no contexto das universidades, oferendo uma visão fundamentada em contextos empíricos aos pesquisadores e empreendedores, aos formuladores de políticas de inovação e demais interessados. 


\section{REFERÊNCIAS}

AMADEI, J.; TORKOMIAN, A. As patentes nas universidades: análise dos depósitos das universidades públicas paulistas. Ciência da Informação, v. 38, n. 2, p. 9-18, 2009.

BARDIN, L. Análise de conteúdo. Lisboa: Edições 70, 1977.

BRASIL. Lei $n .9 .279$, de 14 de maio de 1996. [1996]. Regula a propriedade industrial. Disponível em: <http://www.planalto.gov.br>. Acesso em: 11 out. 2010.

BRASIL. Lei n. 10.406, de 10 de janeiro de 2002. [2002]. Institui o Código Civil brasileiro. Disponível em: <www.planalto.gov.br >. Acesso em: 3 out. 2010.

BRASIL. Lei n. 10.973, de 2 de dezembro de 2004. [2004]. Dispõe sobre incentivos à inovação e à pesquisa científica e tecnológica no ambiente produtivo e dá outras providências. Disponível em: <http://www.planalto.gov.br>. Acesso em: 11 out. 2010.

CGEE - CENTRO DE GESTÃO E ESTUDOS ESTRATÉGICOS. Mestres e doutores 2015: estudos da demografia da base técnico-científica brasileira. Brasília: Centro de Gestão e Estudos Estratégicos, 2016.

CHENG, L.C.; DRUMMOND, P.; MATTOS, P. A integração do trinômio tecnologia, produto e mercado na pré- incubação de uma empresa de base tecnológica. In: Conferência Internacional de Pesquisa em Empreendedorismo na América Latina, 3., 2004, Rio de Janeiro. Anais... Rio de Janeiro: CIPEAL, 2004.

CONDE, Y. C.; SANTOS, M. E. R. Oficinas de transferencia de tecnologia: un analisis comparativo entre las experiencias de una universidad española y outra brasileña. In: Seminario LatinoIberoamericano de Gestión Tecnológica, 8., 1999. Anais... Valencia: ALTEC, 1999.

CUNHA, N. C. V. Mecanismos de interação universidade-empresa e seus agentes: o gatekeeper e o agente universitário de interação. REAd. Revista Eletrônica de Administração, v. 5, p. 1-23, 1999.

DJOKOVIC, D.; SOUITARIS, V. Spinouts from academic institutions: a literatura review with suggestions for further research. Technology Transfer, v. 33, p. 225-247, 2008.

FAVA-DE-MORAES, F. Universidade, inovação e impacto socioeconômico. São Paulo em Perspectiva, v. 14, n. 3, p. 8-11, 2000.

FRANKLIN, S.; WRIGHT, M.; LOCKETT, A. academic and surrogate entrepreneurs in university spinoff companies. Journal of Technology Transfer, v. 26, n. 1-2, p. 127-141, 2001.

GALLON, A. V.; REINA, D. R. M.; ENSSLIN, S. R. O impacto do financiamento da inovação promovido pelo programa juro zero da finep no desempenho econômico-financeiro das MPEIS catarinenses. In: Encontro anual aa Associação Nacional de Pós-Graduação em Administração, 34., 2010, Rio de Janeiro. Anais... Rio de Janeiro: ANPAD, 2010.

GONÇALVES, C. A.; PAIVA Jr., F. G. Competitividade e inovação influenciando o crescimento empresarial: a perspectiva dos empreendedores de empresas de base tecnológica. RPA Brasil, v. 2, p. 55-62, 2006.

HAGE, J.; HOLLINGSWORTH, J. A strategy for the analysis of idea innovation networks and institutions. Organization Studies, v. 21, n. 5, p. 971-1004, 2000.

INPI - INSTITUTO NACIONAL DA PROPRIEDADE INDUSTRIAL. Universidades Brasileiras: Utilização do Sistema de Patentes de 2000 a 2004. Brasília: IBGE, 2007.

INPI - INSTITUTO NACIONAL DA PROPRIEDADE INDUSTRIAL. Principais Titulares de Pedidos de Patente no Brasil: Período de 2004 a 2008. Brasília: INPI, 2011.

JENSEN, M. C.; MECKLING, W. H. The theory of the firm: managerial behavior, agency costs, and capital structure. Journal of Financial Economics, v. 3, n. 4, p. 305-360, 1976. 
LEONEL, S, G. et al. Uma forma de agregar a voz dos clientes nas etapas iniciais de criação de uma empresa de base tecnológica (EBT) de origem acadêmica. In: Simpósio de Gestão da Inovação Tecnológica, 24., 2006, Gramado. Anais... Gramado: ANPAD, 2006.

LOBOSCO, A.; MORAES, M. B.; MACCARI, E. A. Uma análise do papel da agência USP de inovação na geração de propriedade intelectual e nos depósitos de patentes da Universidade de São Paulo. In: Encontro Anual da Associação Nacional de Pós-Graduação em Administração, 34., 2010, Rio de Janeiro. Anais... Rio de Janeiro: ANPAD, 2010.

NDONZUAU, F. N.; PIRNAY, F.; SURLEMONT, B. A stage model of academic spin-off creation. Technovation, v. 22, p. 281-289, 2002.

O'SHEA, R. P.; CHUGH, H.; ALLEN, T. Determinants and consequences of university spinoff activity: a conceptual framework. Technology Transfer, v. 33, p. 653-666, 2008.

PACAGNELLA JR., A. C.; PORTO, G. S.; KANNEBLEY JR., S. A inovação tecnológica nas indústrias do estado de São Paulo: uma análise a partir dos dados da PAEP. In: Encontro Anual da Associação Nacional de Pós-Graduação em Administração, 30., 2006, Salvador. Anais... Salvador: ANPAD, 2006.

PEREIRA, L. B.; MUNIZ, R. M. Obstáculos à inovação: um estudo sobre a geração de spin-offs universitárias na realidade brasileira. In: Simpósio de Gestão da Inovação Tecnológica, 24., 2006, Gramado. Anais... Gramado: ANPAD, 2006.

PROGRAMA de incentivo à inovação. Relatório final da segunda fase do PII na UFLA. Lavras: NTQI, 2008.

PROGRAMA de incentivo à inovação. Relatório final da segunda fase do PII na UFJF. Juiz de Fora: NTQI, 2009.

PROGRAMA de incentivo à inovação. Relatório final da segunda fase do PII na UFMG. Belo Horizonte: NTQI, 2010.

RASMUSSEN, E.; BORCH, O. J. University capabilities in facilitating entrepreneurship: A longitudinal study of spin-off ventures at mid-range universities. Research Policy, v. 39, p. 602-612, 2010.

REIS, L. P. et al. Revelando as fases do planejamento tecnológico no contexto da gestão de desenvolvimento de produtos em empresas iniciantes de base tecnológica. In: Seminário Nacional de Parques Tecnológicos e Incubadoras de Empresas, 16., 2006, Salvador. Anais... Salvador: ANPROTEC, 2006.

REIS, L. P. et al. Proposição de um modelo para o gerenciamento de informações no planejamento tecnológico aplicado a um spinoff do setor de biotecnologia. In: Simpósio de Gestão da Inovação Tecnológica, 31., 2007, Rio de Janeiro. Anais... Rio de Janeiro: ANPAD, 2007.

ROBERTS, E.; MALONE, D. E. Policies and structures for spinning off new companies from research and development organizations. R\&D Management, v. 26, p. 17-48, 1996.

ROBERTS, E. B. Entrepreneurs in high technology: lessons from MIT and beyond. New York: Oxford University Press, 1991.

ROGERS, E.M.; TAKEGAMI, S.; YIN, J. Lesson learned about technology transfer. Technovation, v. 21, p. 253-261, 2001.

RUTHES, S. et al. Inovação tecnológica: um estudo comparativo com os dados da PINTEC. In: Simpósio de Gestão da Inovação Tecnológica, 24., 2006, Gramado. Anais... Gramado: ANPAD, 2006.

SERRA, F. A. R. et al. A inovação numa empresa de base tecnológica: o caso da Nexxera. Journal of Technology Management and Innovation, v. 3, p. 129-141, 2008.

SHANE, S. Academic entrepreneurship, university spinoffs and wealth creation. Cheltenhan, UK: Edward Elgar Publishing, 2004. 
SIEGEL, D. S. et al. Toward a model of the effective transfer of scientific knowledge from academicians to practitioners: qualitative evidence from the commercialization of university tecnologies. Journal Engineering and Technology Management, v. 21, p. 115-142, 2004.

SILVA, S. E.; REIS, L. P. O processo de estruturação de recursos no contexto de uma Empresa de Base Tecnológica de Origem Acadêmica (EBTA). Revista de Administração e Inovação, São Paulo, v. 12, n. 2, p. 153-179, 2015.

TONELLI, D. F.; OLIVEIRA, V. A. R. Os líderes de grupos de pesquisa de uma universidade brasileira legitimam a sua política institucional de inovação? GEPROS - Gestão da Produção, Operações e Sistemas, v. 5, p. 39-51, 2007.

VERGARA, S. C. Projetos e relatórios de pesquisa em administração. 6. ed. São Paulo: Atlas, 2005.

WILLIAMSON, O. E. The theory of the firm as governance structure: from choice to contract. Journal of Economic Prospectives, v. 16, n. 3, p. 171-195, 2002.

WRIGHT, M. et al. Midrange universities linkages with industry: knowledge types and the role of intermediaries. Research Policy, v. 37, p. 1205-1223, 2008. 